\title{
ИНТЕГРАЛЬНЫЕ ПОКАЗАТЕЛИ ДЕМОГРАФИЧЕСКИХ ПОТЕРЬ ОТ СМЕРТНОСТИ И ТРАВМАТИЗМА В РЕЗУЛЬТАТЕ ДОРОЖНО-ТРАНСПОРТНЫХ ПРОИСШЕСТВИЙ В РОССИИ *
}

\author{
АЛЕКСАНДР РАМОНОВ
}

\begin{abstract}
В работе выполнен анализ динамики и структуры смертности и травматизма от дорожнотранспортных происшествий (ДТП) в России с 2006 по 2011 2., основанный на использовании интегральных показателей потерянных лет жизни. Показано, что максимальное число лет жизни, потерянных в результате смертности и травматизма (или выцгранных в результате их устранения), среди мужчин приходится на возрастные группь от 40 до 65 лет, среди женщин - на промежуток от 55 до 75 лет.
\end{abstract}

Применен новый метод оценки тяжести повреждений, наступивших в результате ДТП и других внешних причин.

Ключевые слова: внешние причины смерти, интегральные показатели здоровья, дорожнотранспортные происшествия.

Эпидемиология внешних причин смерти (The epidemiology of accident traumas and resulting disabilities) - направление эмпирических исследований, посвященное изучению совокупности средовых и поведенческих факторов и последствий внешних причин в виде смертности и травматизма на основе инструментария и показателей, разработанных в рамках эпидемиологии и демографии. Одно из первых его упоминаний связано с публикацией отчета регионального европейского бюро ВОЗ в 1981 г. [WHO... 1981], посвященного изучению последствий травматизма (в первую очередь в результате транспортных несчастных случаев) в европейских странах. Там же разработана классификация системы факторов (средовых и индивидуальных), увеличивающих риск попасть в происшествие (или оказаться его инициатором). Специфика данного направления по сравнению с классической эпидемиологией заключается в том, что «возбудитель» и «носитель» априори неразделимы и воплощены в действующих участниках, помещенных в материальное и социальное окружение. Условными «носителями» также могут оказаться сами предметы материального окружения (например, в случае неисправности транспортных средств в дорожно-транспортных происшествиях - ДТП). Это требует особой внимательности от политиков и экспертов при разработке комплексных решений, направленных на снижение смертности и травматизма в результате этих причин.

\footnotetext{
АЛЕКСАНДР ВЛАДИМИРОВИЧ РАМОНОВ. НАЦИОНАЛЬНЫЙ ИССЛЕДОВАТЕЛЬСКИЙ УНИВЕРСИТЕТ «ВЫСШАЯ ШКОЛА ЭКОНОМИКИ» (РосСИЯ). E-mail: aramonov@hse.ru

СТАТЬЯ ПОСТУПИЛА В РЕДАКЦИЮ В ОКТЯБРЕ 2015 Г.

* СТАТЬЯ ПОДГОТОВЛЕНА ПО РЕЗУЛЬТАТАМ ИССЛЕДОВАНИЯ 15-01-ОО32, ВЫПОЛНЕННОГО В РАМКАХ ПРОГРАММЫ «НАУЧНЫЙ ФОНД НАЦИОНАЛЬНОГО ИССЛЕДОВАТЕЛЬСКОГО УНИВЕРСИТЕТА «ВЫСШАЯ ШКОЛА ЭКОНОМИКИ» (НИУ ВШЭ)» В 2015 Г. И С ИСПОЛЬЗОВАНИЕМ СРЕДСТВ СУБСИДИИ НА ГОСУДАРСТВЕННУЮ ПОДДЕРЖКУ ВЕДУЩИХ УНИВЕРСИТЕТОВ РОССИЙСКОЙ ФЕДЕРАЦИИ В ЦЕЛЯХ ПОВЫШЕНИЯ ИХ КОНКУРЕНТОСПОСОБНОСТИ СРЕДИ ВЕДУЩИХ МИРОВЫХ НАУЧНО-ОБРАЗОВАТЕЛЬНЫХ ЦЕНТРОВ, ВЫДЕЛЕННОЙ НИУ ВШЭ.
} 
Оценка интегральных потерь (выигрыша от устранения) в результате воздействия внешних причин является относительно новым витком исследований в рамках этого направления, толчок которому дало проведение первого международного исследования «Глобальное бремя болезней» (ГББ) в 1990 г. Интегральные показатели потерь населения или выигрыша в результате устранения, все шире используемые в практике социальнодемографического анализа, позволили произвести комплексную оценку демографических потерь: потерь лет жизни в результате смертности и лет здоровой жизни в результате нарушений здоровья, связанных с полученными травмами. В плане оценки демографических последствий внешних причин смерти примечательна попытка оценить демографические потери в результате транспортных несчастных случаев (Глобальное бремя транспортного травматизма), предпринятая Всемирным банком по результатам международного исследования ГББ-2010, инициированного Всемирной организацией здравоохранения [The World Bank 2014]. По сделанным оценкам, транспортные несчастные случаи в 2010 г. (единственная причина из класса «Внешние причины смерти», которая вошла в ТОП-10 ведущих причин потерь) занимали восьмое место в мире по количеству лет жизни, потерянных в результате смертности (1,3 млн), и лет здоровой жизни, потерянных в результате травматизма (74,2 млн) - индекс DALYs (Disability Adjusted Life Years).

Учитывая масштабы самого феномена травматизма в результате воздействия внешних причин, оценка их демографических последствий представляет актуальную задачу. К сожалению, решение этой задачи наталкивается на серьезные информационные и методологические трудности.

Новизна данной работы состоит в том, что обоснованы применимость и выбор данных о регистрируемом травматизме для оценки его масштабов в результате ДТП и усовершенствована методология их расчета.

\section{ИСТОЧНИКИ ДАННЫХ О ТРАВМАТИЗМЕ}

Для оценки демографических потерь от смертности и травматизма в исследовании Глобального бремени транспортного травматизма в 2010 г. была применена многоступенчатая методология, основанная на комбинированном использовании показателей из нескольких источников данных. Распространенность транспортных происшествий оценивалась на основе выборочных обследований населения (микроданные), последствия от полученных травм для здоровья и жизнедеятельности - на основе данных медицинских учреждений, средняя длительность пребывания в состояниях нездоровья - на основе выборочных обследований пациентов.

К сожалению, далеко не все страны располагают дифференцированными по возрасту показателями, необходимыми для оценки транспортной смертности и травматизма, оценки, сделанные в докладе, основаны на модельных построениях для регионов мира. Россия в соответствии с классификацией ГББ не относится к странам с достоверной дифференцированной по возрасту статистикой смертности и травматизма для исчисления надежных показателей потерь. Тем не менее в России имеются некоторые источники 
данных, позволяющих оценить интегральные потери в результате воздействия ряда внешних причин. Это как информация о регистрируемых случаях травматизма, так и данные, полученные со слов респондентов из выборочных обследований.

Регистрируемые случаи травматизма как причины потерь здоровья находят отражение в нескольких формах статистической отчетности:

- форма №16-ВН - заболеваемость с временной утратой трудоспособности (подотчетное ведомство - Минздрав);

- форма №7-собес - первичная инвалидность (подотчетные ведомства - Минздрав, бюро МСЭ);

- форма №57 - по данным об обращаемости в амбулаторно-поликлинические учреждения и по итогам стационарного лечения (подотчетное ведомство - Минздрав).

Показатели, полученные на основе формы №16-BH «О причинах временной нетрудоспособности», содержат информацию о случаях утраты трудоспособности по полу и возрасту (по 5-летним группам в интервале от 15 до 39 лет) по классам причин и характеру полученных травм (коды групп причин $\mathrm{S}$ и Т по МКБ-10). Согласно методике сбора данных, предусмотренной формой, единицей наблюдения является «круглогодовой рабочий» (работающий на предприятии не менее года).

Форма №7-собес содержит результаты первичных освидетельствований в бюро МСЭ, на основе которых устанавливаются причины первичной инвалидности (по МКБ-10). В ней эти показатели даны в разбивке по укрупненным интервалам: дети до 18 лет, трудоспособное население, население старше трудоспособного.

Форма №57 - единственная из вышеперечисленных имеет специализированный характер, поскольку по ней регистрируются случаи оказания медицинской помощи только при указании в качестве причины одной из группь «внешние причины смерти». Форма также содержит информацию о регионе, месте происшествия и характере полученных травм и ранений (более подробно ее структура дана ниже).

Информащию о распространенности травматизма по самооценке дают выборочные обследования. Наиболее крупные из них, содержащие вопросы о последствиях травм: World Health Survey (проведено в России в 2003 г.) и WHO SAGE (первая волна в России проведена в 2007-2010 гг.). Они позволяют оценить распространенность травматизма за год, предшествующий наблюдению, со слов респондентов на основании соответствующих вопросов анкеты. Однако в выборках соответствующих обследований крайне незначительно представлены те, кто получил нарушения здоровья в результате воздействия внешних факторов ${ }^{1}$. Это затрудняет дальнейший подробный анализ по полу и возрасту. Поэтому этот источник данных представляет небольшую ценность в рамках настоящего исследования.

\footnotetext{
${ }^{1}$ По данным WHO SAGE наиболее распространенными среди причин получения травм и ранений в 2007-2010 гг. были указаны: случайные падения - в 119 случаях, ДТП - в 98 случаях при общем объеме выборки 3418 респондентов.
} 
Для оценки распространенности травматизма использована форма регистрируемых случаев травматизма № 57, поскольку она единственная из представленных покрывает все население России, а не только его трудоспособную (форма №16-ВН) или обратившуюся в органы социальной защиты (№7-собес) группы. Также она единственная содержит указание не только на характер полученной травмы, но и на место и обстоятельство происшествия, в частности, позволяет выделить «Транспортный несчастный случай» и «ДТП» как причины полученной травмы, что представляет ценную информацию при расчете интегральных потерь от этих причин.

В распоряжении имелись данные о травматизме, полученные и агрегированные по форме №57 в России в целом за период наблюдения с 2006 по 2011 г.

Форма содержит сведения о случаях полученных травм и ранений в результате воздействия внешних причин и оказания впоследствии медицинской помощи. Она составляется всеми медицинскими организациями и подразделениями, оказывающими медицинскую помощь пострадавшим от травм, отравлений и других последствий воздействия внешних причин, на основе двух первичных учетных форм:

- талона амбулаторного пациента;

- статистической карты выбывшего из стационара.

Форма №57 состоит из таблиц, включающих сведения о травмах, отравлениях и внешних причинах у детского (до 14 лет) и взрослого (15+) населения. Общее количество травм получено как сумма травм среди взрослых старше 15 лет (связанных и не связанных с производством) и детей (0-14 лет).

\section{РАСПРОСТРАНЕННОСТЬ И СТРУКТУРА ТРАВМАТИЗМА В РОССИИ}

В России ежегодно общее количество полученных мужчинами и женщинами травм в результате воздействия внешних причин составляет порядка 13 млн, примерно 60\% из них приходится на мужчин (рисунок 1). С 2006 по 2011 г. общее количество травм у мужчин осталось примерно на одном уровне, у женщин выросло на 9\%.

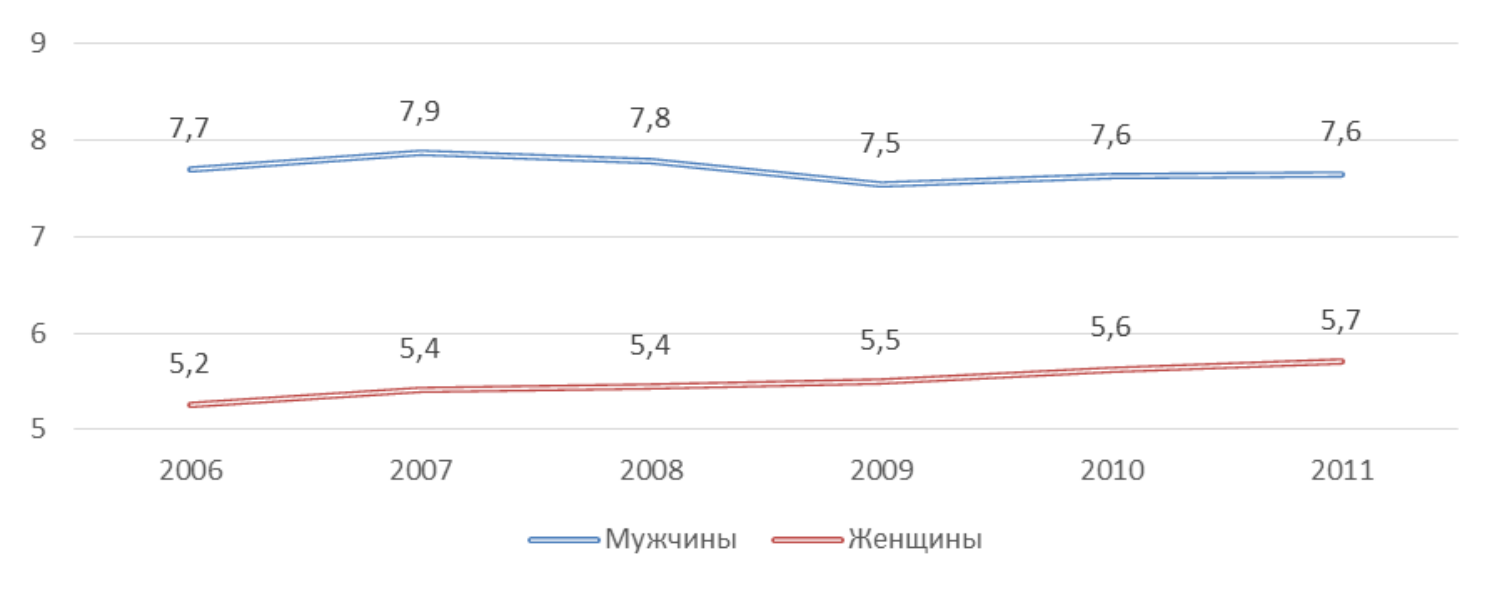

Рисунок 1. Общее число полученных травм, 2006-2011, млн

Источник: Данные Минздрава, полученные по форме статистического наблюдения №57. 
Далее при изучении структуры травматизма рассмотрены его усредненные показатели, рассчитанные на основе данных 2006-2011 гг., которые дают наглядное представление о структуре полученных травм по возрасту и их локализации.

Основное количество травм пришлось на население старше 18 лет - порядка 9,3 млн из общего количества 12,9 млн.

Таблица 1 и рисунок 2 дают представление об объеме и структуре травматизма в России.

Таблица 1. Количество травм, полученных мужчинами и женщинами с 2006 по 2011 г., в среднем за год в России по видам полученных травм, тыс. случаев

\begin{tabular}{|c|c|c|}
\hline Вид травм & Мужчины & Женщины \\
\hline Поверхностные травмы & 2373,4 & 1775,9 \\
\hline Открытые раны, травмы кровеносных сосудов & 1532,9 & 862,3 \\
\hline Вывихи, травмы мышц и сухожилий & 845,1 & 703,2 \\
\hline Переломы костей верхней конечности & 784,2 & 638,9 \\
\hline Переломы костей нижней конечности & 555,0 & 404,3 \\
\hline Внутричерепные травмы & 321,4 & 198,5 \\
\hline Переломы позвоночника, костей туловища & 202,9 & 103,8 \\
\hline Термические и химические ожоги & 201,9 & 149,8 \\
\hline Травмы глаза и глазницы & 136,1 & 57,4 \\
\hline Последствия травм, отравлений, других воздействий внешних причин & 132,2 & 70,8 \\
\hline Переломы черепа и лицевых костей & 113,1 & 42,4 \\
\hline Другие & 496,8 & 242,3 \\
\hline Всего & 7695,1 & 5250,0 \\
\hline
\end{tabular}

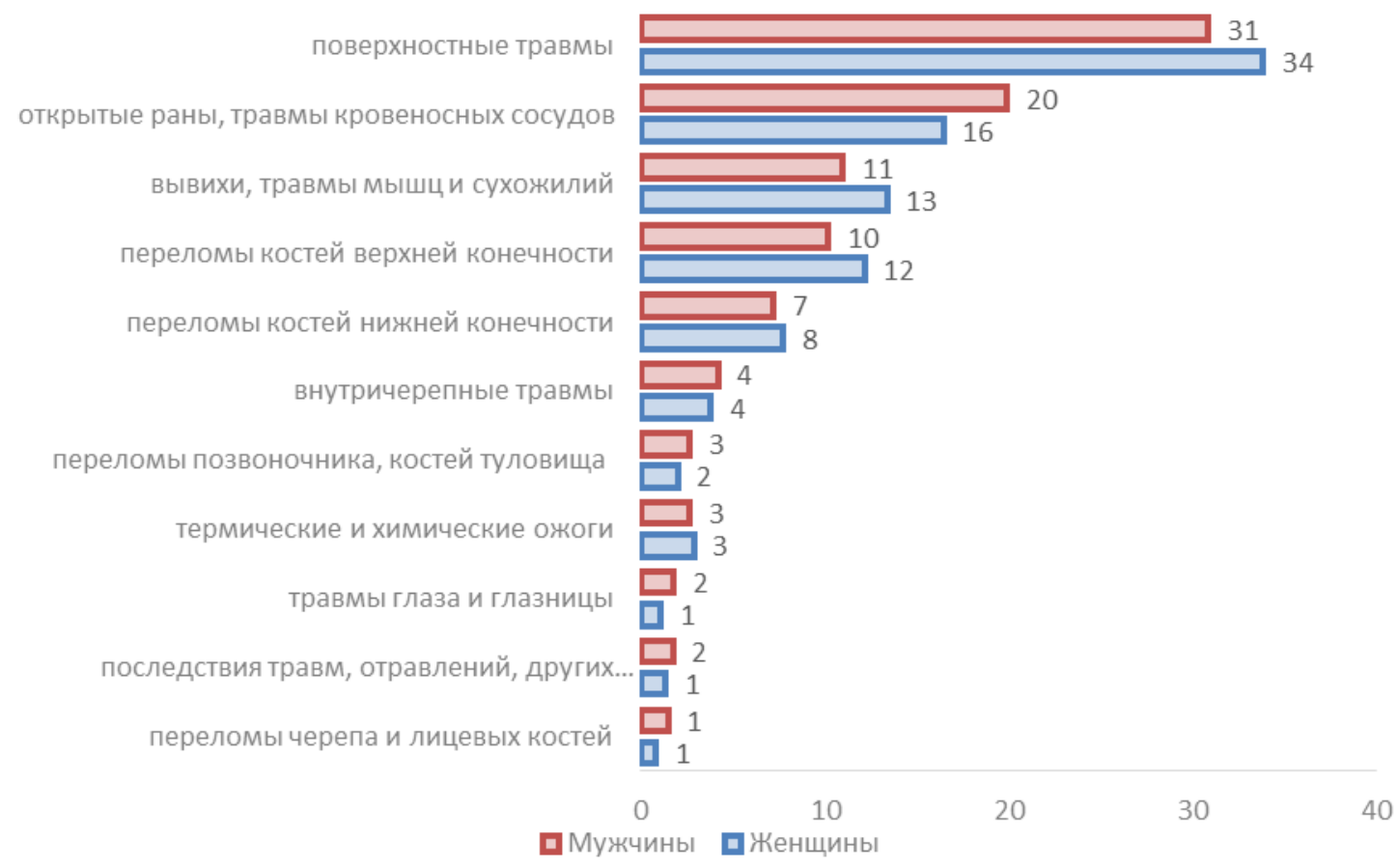

Рисунок 2. Распределение общего количества травм, полученных мужчинами и женщинами с 2006 по 2011 г. в России, в среднем за год, \%

Источник: Данные Минздрава, полученные по форме статистического наблюдения №57. 
Поверхностные раны и травмы кровеносных сосудов, как правило, имеют незначительные последствия для организма и функционирования его отдельных систем в отличие от переломов костей и травм внутренних органов.

\section{ТРАВМАТИЗМ В РЕЗУЛЬТАТЕ ДТП}

Согласно информации по форме статистического наблюдения №57 с 2006 по 2011 г. в среднем было зафиксировано 180,5 тыс. травм, наступивших в результате ДТП, 87\% которых пришлось на взрослое население (15+). 61\% общего количества полученных в результате ДТП травм пришелся на мужчин.

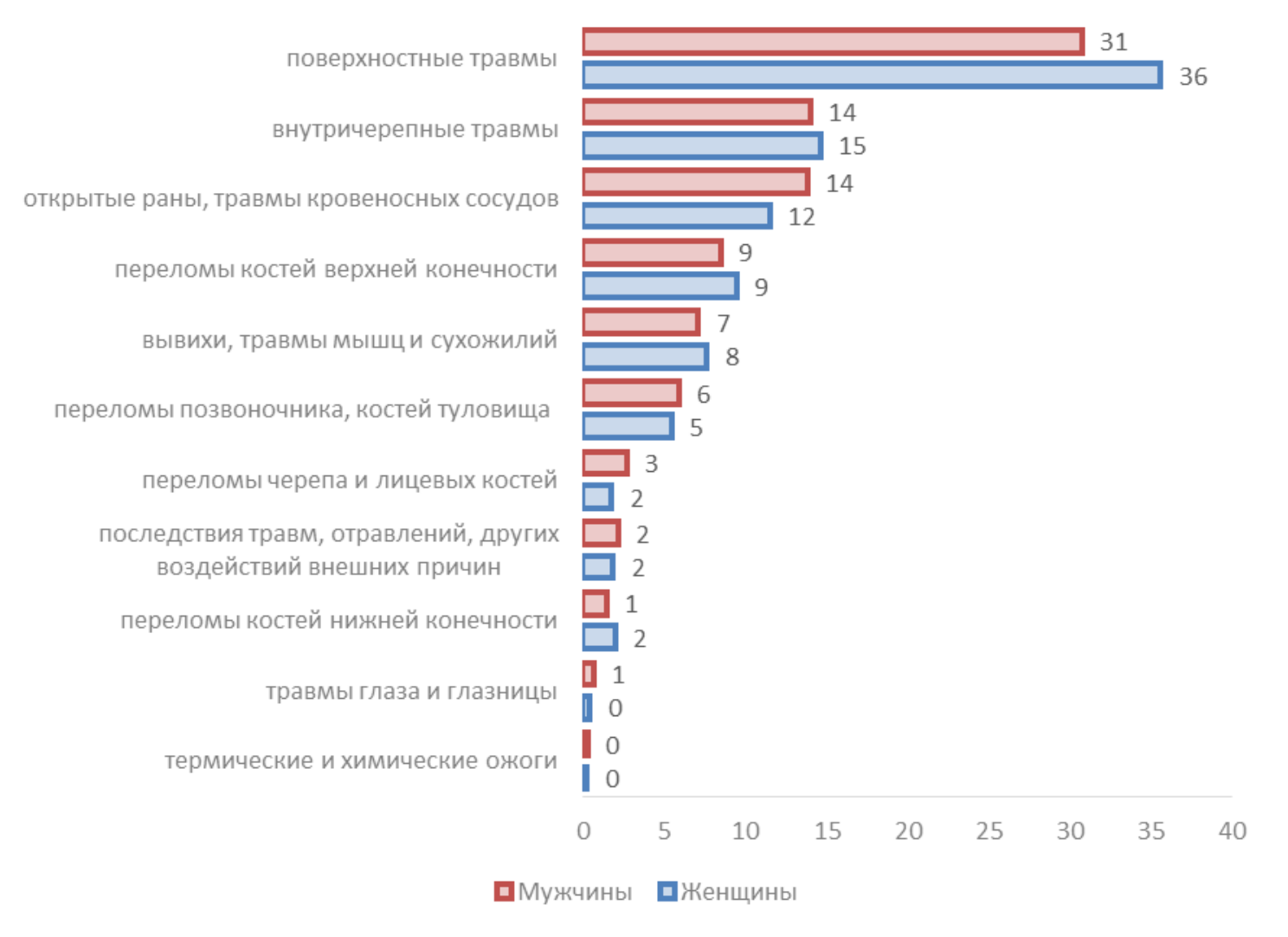

\section{Рисунок 3. Доля травм отдельных локализаций в общем объеме травматизма в результате ДТП с 2006 по 2011 гг., в среднем за год, \%}

Источник: Данные Минздрава, полученные по форме статистического наблюдения №57.

По сравнению со структурой общего травматизма (рисунок 2) в структуре дорожнотранспортного травматизма можно наблюдать более высокие значения распространенности внутричерепных травм (рисунок 3 ).

Для оценки демографических потерь использованы данные о взрослом населении (от 15 лет), поскольку именно в этих группах сосредоточены основные объемы смертности и травматизма как у мужчин, так и у женщин. Всего среди взрослого населения 15+ было получено в результате ДТП 96,2 тыс. травм мужчинами и 60,2 тыс. травм женщинами. 
Таблица 2. Числа смертей и травм в результате ДТП среди населения в возрасте 15 лет и старше, распределенные по пятилетним возрастным группам, 2006-2011, в среднем за год

\begin{tabular}{l|c|c|c|c}
\hline \multirow{2}{*}{ Возраст, лет } & \multicolumn{2}{|c|}{ Травмы } & \multicolumn{2}{c}{ Смерти } \\
\cline { 2 - 5 } & мужчины & женщины & мужчины & женщины \\
\hline $15-19$ & 5753 & 4793 & 1387 & 658 \\
$20-24$ & 13740 & 6363 & 3311 & 873 \\
$25-29$ & 13335 & 5370 & 3214 & 619 \\
$30-34$ & 11108 & 4510 & 2677 & 570 \\
$35-39$ & 9118 & 4156 & 2198 & 550 \\
$40-44$ & 8701 & 4008 & 2097 & 671 \\
$45-49$ & 9303 & 4887 & 2242 & 673 \\
$50-54$ & 8174 & 4903 & 1970 & 560 \\
$55-59$ & 5948 & 4083 & 1434 & 377 \\
$60-64$ & 3155 & 2749 & 760 & 583 \\
$65-69$ & 2758 & 3519 & 665 & 501 \\
$70-74$ & 2445 & 4071 & 589 & 316 \\
$75-79$ & 1634 & 3647 & 394 & 111 \\
$80-84$ & 809 & 2301 & 195 & 8258 \\
$85+$ & 250 & 810 & 60 & \\
Всего & 96232 & 60170 & 23192 & \\
\hline
\end{tabular}

Прежде чем использовать эти данные наравне со статистикой смертности для оценки выигранных лет жизни с помощью таблиц смертности, необходимо оценить их возрастные распределения. Одним из возможных вариантов их построения является использование возрастного распределения чисел умерших для соответствующей группировки общего числа полученных травм среди населения $15+^{2}$. Результаты подобной группировки представлены в таблице 2 и на рисунке 4.

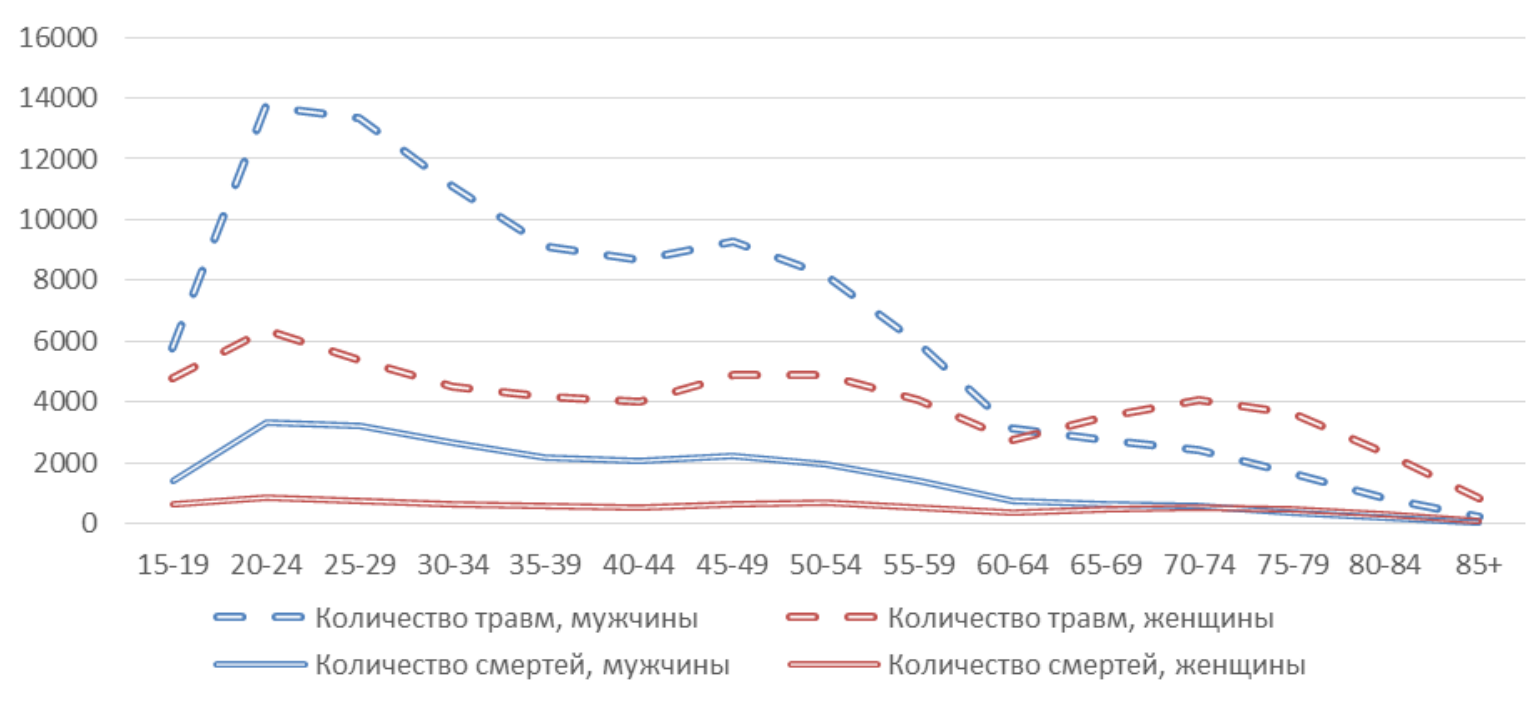

\section{Рисунок 4. Числа смертей и травм в результате ДТП в России, распределенные по пятилетним возрастным группам, 2006-2011, в среднем за год}

Источник: Расчеты автора на основе данных Минздрава, полученных по форме статистического наблюдения №57 и данных о регистрачии смертей, Росстат.

\footnotetext{
${ }^{2}$ Подобное распределение предполагает, что летальность ДТП (соотношение смертей и травм) в исходных данных с возрастом не меняется.
} 


\section{ВЫИГРЫШ ЛЕТ ЖИЗНИ И ЗДОРОВОЙ ЖИЗНИ В РЕЗУЛЬТАТЕ УСТРАНЕНИЯ ДТП в РОССИИ}

\section{Выигрыш лет жсизни в результате устранения смертности}

При расчете потерянных лет жизни в результате смертности от определенных причин (или выигранных лет в результате устранения) в качестве базы для сравнения принято использовать определенный стандарт - обычно это страна или модельный образец страны с низкой смертностью [Murray, Lopez 1997]. Здесь применен альтернативный подход, в рамках которого выигрыш лет жизни рассчитывается по сравнению с гипотетической ситуацией полного устранения смертности от этой причины. В отношении ДТП такое предположение является достаточно правдоподобным.

Для расчета показателей по этому методу необходимо отнять от возрастных показателей общей смертности $m(x)$ аналогичные показатели смертности вследствие ДТП $m(x)_{t}$ и рассчитать новые вероятности умереть и другие функции таблицы смертности. Перерасчет вероятности умереть для таблицы смертности при устранении ДТП осуществлен следующим способом:

$$
g(x)_{t-}=\frac{2 n\left(m(x)-m(x)_{t}\right)}{2+n\left(m(x)-m(x)_{t}\right)}
$$

где $n$ - длина возрастного интервала (в данном случае равна 5), $m(x)$ - возрастные коэффициенты общей смертности, $m(x)_{t}$ - возрастные коэффициенты смертности в результате ДТП.

Таблица 3. Некоторые показатели таблицы смертности в целом и при устранении смертности в ДТП для женщин в России, 2006-2011, в среднем за год

\begin{tabular}{|c|c|c|c|c|c|c|c|c|c|}
\hline $\begin{array}{l}\text { Россия, } \\
\text { женщины, } \\
\text { 2006-2011 гг., } \\
\text { в среднем }\end{array}$ & \multicolumn{4}{|c|}{ Общая смертность } & \multicolumn{4}{|c|}{ Смертность при устранении ДТП } & Выигрыш \\
\hline$x$ & $m(x)$ & $q(x)$ & $L(x)$ & $T(x)$ & $m(x)_{t-}$ & $q(x)_{t-}$ & $L(x)_{t-}$ & $T(x)_{t-}$ & $T(x)_{t-}-T(x)$ \\
\hline $15-19$ & 0,001 & 0,003 & 499228 & 6003839 & 0,000 & 0,002 & 499399 & 6027629 & 23790 \\
\hline $20-24$ & 0,001 & 0,004 & 497389 & 5504611 & 0,001 & 0,004 & 497908 & 5528230 & 23619 \\
\hline $25-29$ & 0,001 & 0,007 & 494599 & 5007222 & 0,001 & 0,006 & 495447 & 5030322 & 23100 \\
\hline $30-34$ & 0,002 & 0,010 & 490455 & 4512623 & 0,002 & 0,009 & 491592 & 4534875 & 22253 \\
\hline $35-39$ & 0,003 & 0,012 & 484993 & 4022168 & 0,002 & 0,012 & 486397 & 4043283 & 21115 \\
\hline $40-44$ & 0,003 & 0,016 & 478004 & 3537175 & 0,003 & 0,016 & 479655 & 3556886 & 19711 \\
\hline $45-49$ & 0,005 & 0,022 & 468747 & 3059171 & 0,004 & 0,022 & 470622 & 3077230 & 18059 \\
\hline $50-54$ & 0,006 & 0,032 & 456048 & 2590425 & 0,006 & 0,031 & 458125 & 2606609 & 16184 \\
\hline $55-59$ & 0,010 & 0,048 & 437875 & 2134377 & 0,010 & 0,048 & 440107 & 2148484 & 14107 \\
\hline $60-64$ & 0,013 & 0,065 & 413173 & 1696502 & 0,013 & 0,065 & 415505 & 1708377 & 11875 \\
\hline $65-69$ & 0,021 & 0,102 & 378891 & 1283329 & 0,021 & 0,101 & 381258 & 1292872 & 9542 \\
\hline $70-74$ & 0,034 & 0,157 & 330378 & 904438 & 0,034 & 0,156 & 332674 & 911613 & 7175 \\
\hline $75-79$ & 0,061 & 0,264 & 262402 & 574060 & 0,061 & 0,263 & 264439 & 578939 & 4879 \\
\hline $80-84$ & 0,101 & 0,405 & 177542 & 311659 & 0,101 & 0,404 & 179072 & 314500 & 2842 \\
\hline $85+$ & 0,198 & 1 & 134117 & 134117 & 0,197 & 1 & 135428 & 135428 & 1311 \\
\hline
\end{tabular}

Далее на основе моделирования дожития условной когорты оценивается количество человеко-лет, прожитых населением в каждой возрастной группе, которое рассчитывается по стандартным, принятым в демографии формулам расчета числа живущих отдельно для 
реальной $(L(x))$ и гипотетической ситуации при устранении смертности в ДТП - $L(x)_{t}$ (начальная численность условной когорты принята за 100000).

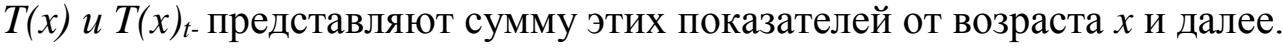

Пример расчета совокупного выигрыша женщин продемонстрирован в таблице 3.

Распределенные по возрасту числа выигранных человеко-лет представлены на рисунке 5 .

Совокупный выигрыш мужчин с 2006 по 2011 г. в среднем составил 56353 человеколет, женщин - 23790 человеко-лет.

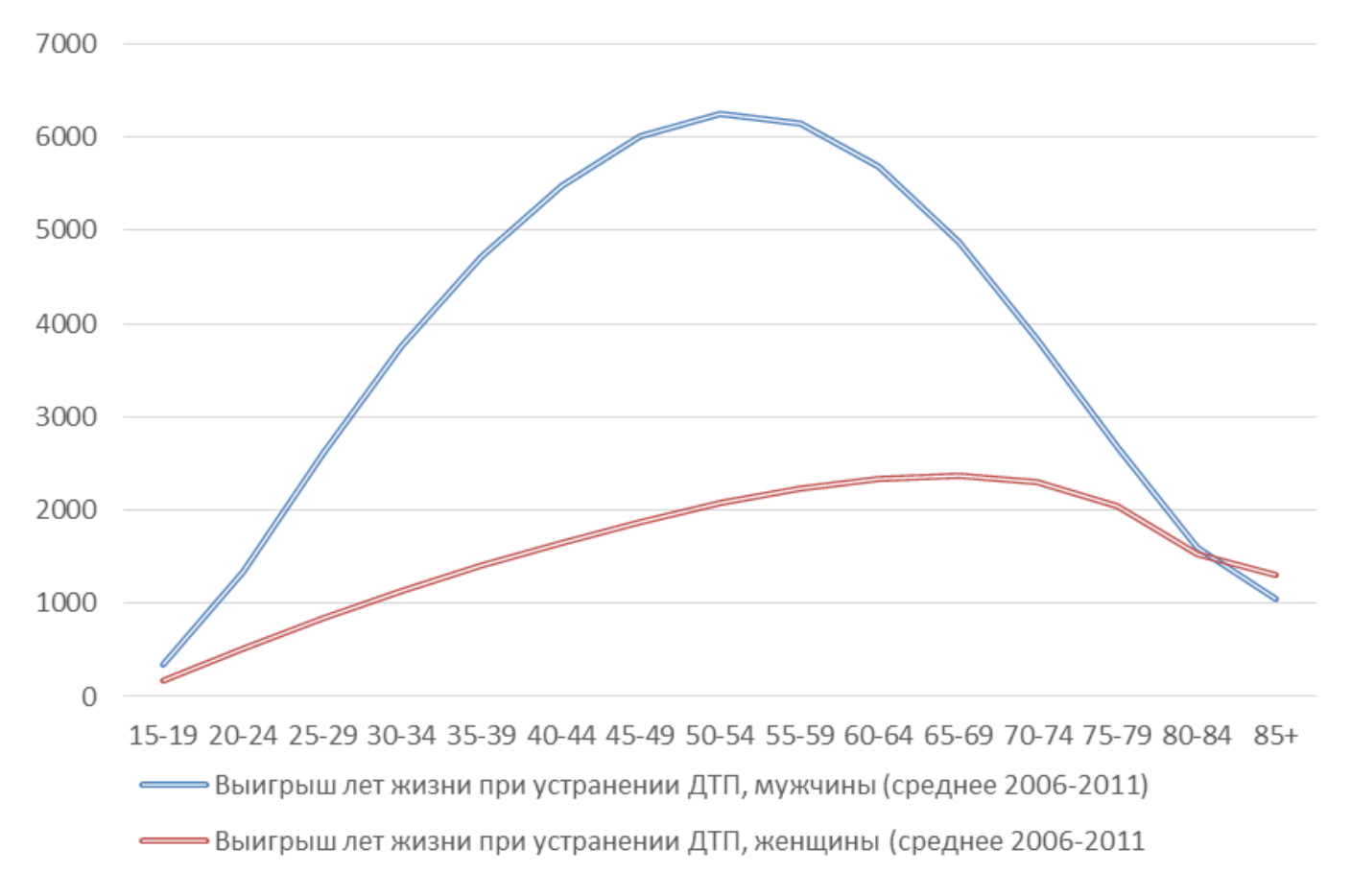

Рисунок 5. Годы предстоящей жизни, выигранные в результате устранения смертности от ДТП в России с 2006 по 2011 г., в среднем за год, население 15 лет и старше

Источник: Расчеты автора на основе данных Минздрава, полученных по форме статистического наблюдения №57 и данных о регистрации смертей, Росстат.

Как видно из рисунка 5, наибольшие потери предстоящих лет жизни у мужчин достигаются в старших трудоспособных возрастах, тогда как у женщин - в пожилых, в которых максимум достигается только в возрастах 65-69 лет.

\section{Выигрыш времени здоровой жсизни в результате устранения травматизма}

Для расчета выигрыша, выраженного в годах здоровой жизни, необходимо сначала соотнести возрастные числа травм из таблицы 2 с усредненной за соответствующие годы (2006-2011 гг.) численностью населения - тем самым получить значения распространенности травматизма или коэффициенты травматизма $\pi(x)$. 
В рамках модели множественного выбытия (multistate life table), показатели которой для случая ДТП представлены в таблице 4, предполагается, что из общего числа человеколет таблицы смертности определенное число выбывает в результате смертности в ДТП. Оставшиеся представители населения $(L(x))^{\prime}$ - общее число лет, прожитых ими в интервале $x$ ) подвержены риску получить ранения в результате ДТП и/или умереть от других причин смерти. $L H_{-}(x)^{\prime}-$ число человеко-лет, прожитых при наличии травм среди тех, кто не умер в результате ДТП в данном возрастном интервале (рисунок 6).

Таблица 4. Некоторые показатели мультистатусной таблицы смертности женщин в России, 2006-2011, в среднем за год

\begin{tabular}{l|c|c|c}
\hline$x$ & $\pi(x)$, на 100000 человек населения & $L(x)^{\prime}$ & $L H_{-}(x)^{\prime}$ \\
\hline $15-19$ & 100 & 499228 & 1075 \\
$20-24$ & 104 & 497389 & 3258 \\
$25-29$ & 92 & 494599 & 5330 \\
$30-34$ & 84 & 490455 & 7151 \\
$35-39$ & 84 & 484993 & 8830 \\
$40-44$ & 79 & 478004 & 10382 \\
$45-49$ & 80 & 468747 & 11788 \\
$50-54$ & 81 & 456048 & 13055 \\
$55-59$ & 77 & 437875 & 14035 \\
$60-64$ & 82 & 413173 & 14663 \\
$65-69$ & 94 & 378891 & 14881 \\
$70-74$ & 112 & 330378 & 14434 \\
$75-79$ & 130 & 262402 & 12807 \\
$80-84$ & 118 & 177542 & 9621 \\
$85+$ & 87 & 134117 & 8243 \\
\hline
\end{tabular}

25000

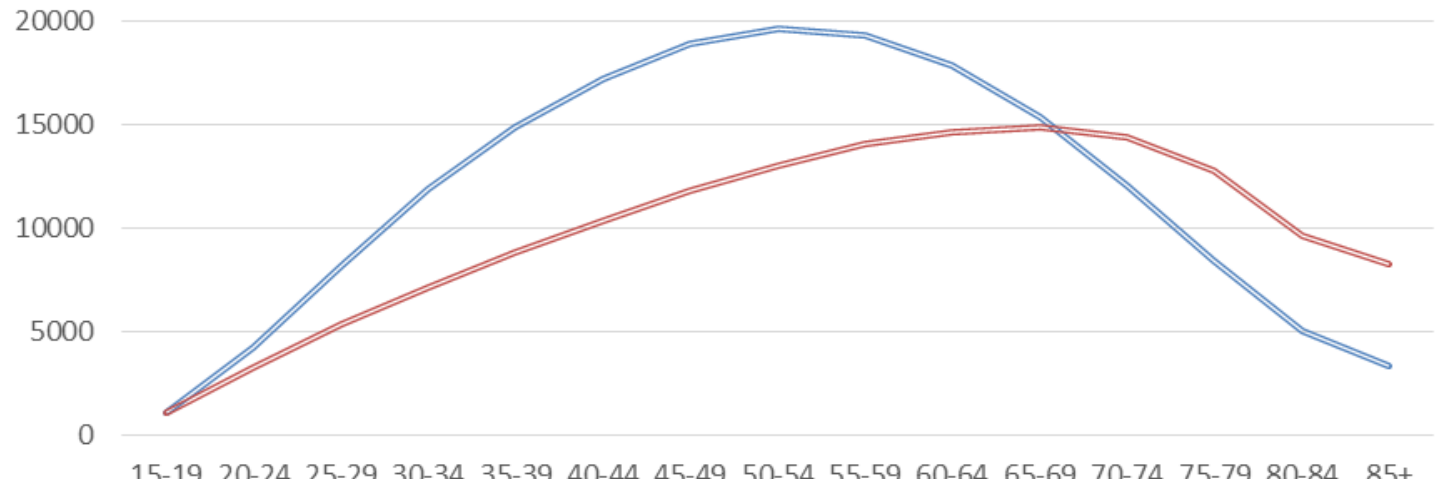

15-19 20-24 25-29 30-34 35-39 40-44 45-49 50-54 55-59 60-64 65-69 70-74 $75-79 \quad 80-84 \quad 85+$

ऍ Годы, прожитые при наличии травм, мужчины 2006-2011

ऍ Годы, прожитые при наличии травм, женщины 2006-2011

Рисунок 6. Годы нездоровой жизни, прожитые при наличии травм (или годы здоровой жизни, выигранные от устранения травм), полученных в результате ДТП в России с 2006 по 2011 г., в среднем за год, население 15 лет и старше

Источник: Расчеты автора на основе данных Минздрава, полученных по форме статистического наблюдения №57 и данных о регистрачии смертей, Росстат.

В продемонстрированной методологии расчета потерь в результате ДТП сделано два допущения. 
1. Год жизни, потерянный в результате травматизма, приравнивается к потерянному году жизни в результате смертности в рамках таблиц смертности для населения в целом. Как было показано раннее, последствия травматизма могут быть различными, во многих случаях вообще не приводить к ограничениям в жизнедеятельности. Ввиду этого необходима дальнейшая корректировка полученных значений на степень тяжести ограничений ${ }^{3}$.

2. По умолчанию принимается, что длительность периода нетрудоспособности, наступившего в результате травмы, равна половине длительности возрастного интервала - 2,5 года. Для более достоверной оценки необходимо иметь представление о периоде жизни, проживаемом с ограничениями среди представителей различных половозрастных групп.

Далее решена первая задача на основе информации, которой располагают исследователи в рамках формы №57. Решение второй задачи на данном этапе представляется невозможным ввиду отсутствия статистических данных.

\section{Корректировка на степень тяжести полученных травм}

Не все полученные травмы приводят к серьезным функциональным нарушениям. Для более достоверной оценки необходимо определить виды травм, которые влияют на трудоспособность. Далее произведена попытка решить эту задачу с помощью введения функции весов тяжести последствий полученных травм (Disability weights или $D w$ ), использовав для ее построения данные о локализации травм по XIX классу МКБ-10.

В качестве локализаций травм, связанных с тяжелыми ограничениями и длительной утратой трудоспособности, определены травмы, связанные с переломами костей и нарушением функционирования внутренних органов:

- внутричерепные травмы;

- переломы костей нижней конечности;

- переломы костей верхней конечности;

- вывихи, растяжения и перенапряжения капсульно-связочного аппарата суставов, травмы мышц и сухожилий;

- переломы позвоночника, костей туловища, других и неуточненных областей тела;

- переломы черепа и лицевых костей;

- последствия травм, отравлений, других воздействий внешних причин;

- травмы внутренних органов грудной и брюшной областей, таза;

- размозжения (раздавливание), травматические ампутации;

- травмы глаза и глазницы;

- травмы нервов и спинного мозга;

- термические и химические ожоги.

\footnotetext{
${ }^{3}$ В рамках исследования ГББ это сделано с помощью специально разработанной системы весов для каждой причины, оцениваемых методом экспертных оценок.
} 
Доля травм этих видов в общем количестве автодорожных травм мужчин 15+ в 2006-2011 гг. в среднем составила 43\%, у женщин - 44\%.

Скорректировав общее количество лет жизни, прожитых при наличии травм, на их тяжесть, можно рассчитать общее количество лет, потерянное только в связи с тяжелыми травмами (или выигранное от их устранения), которое у мужчин составило 76316 человеколет, у женщин - 65803 человеко-лет.

Сводная картина полученных показателей в России с 2006 по 2011 г. в среднем в разбивке по полу представлена на рисунке 7. Интересно, что среди женщин вклад травматизма в общий объем потерянных лет в результате ДТП оказался значимо выше, чем среди мужчин (73\% против 58\%).

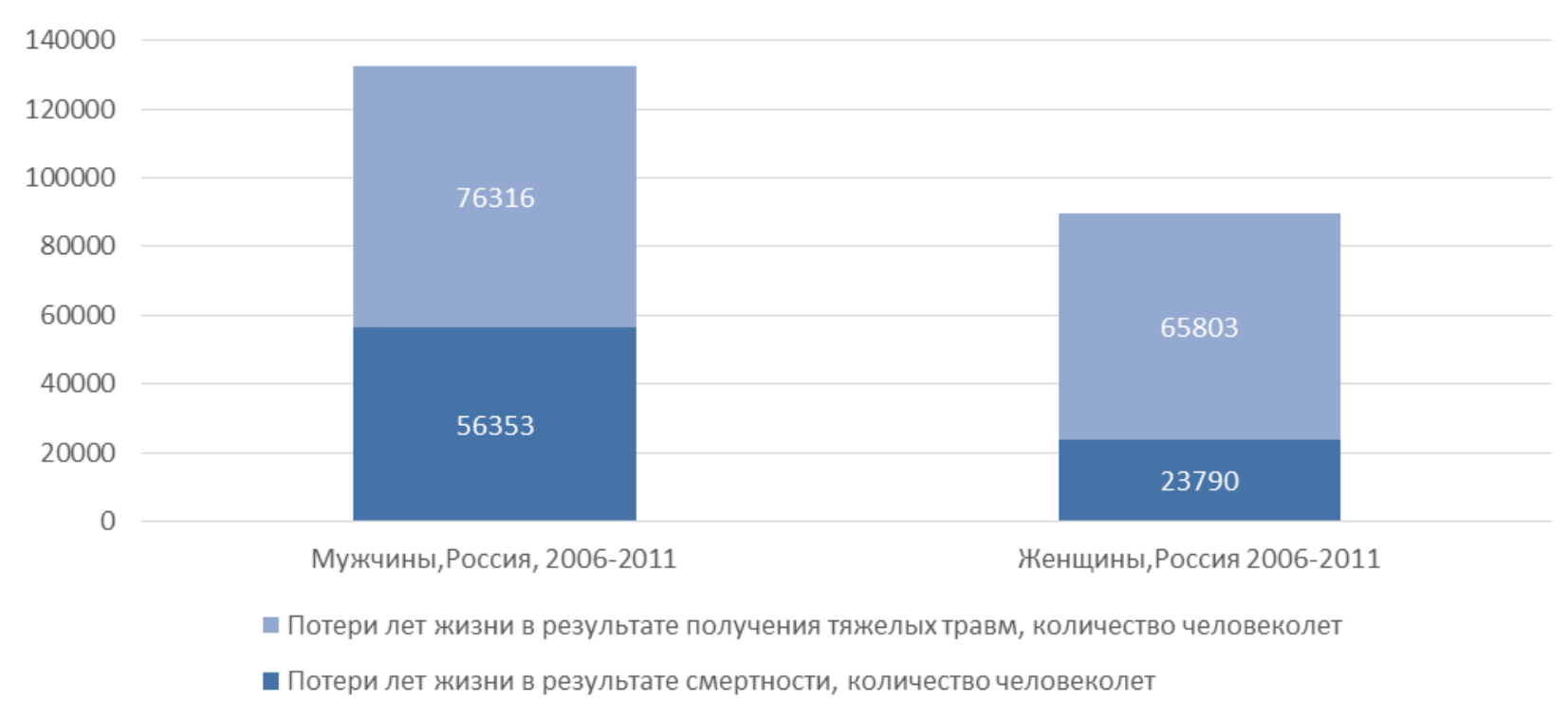

\section{Рисунок 7. Годы жизни, потерянные в результате ДТП в России с 2006 по 2011 г., в среднем за год, население 15 лет и старше}

Источник: Расчеты автора на основе данных Минздрава, полученных по форме статистического наблюдения №57 и данных о регистрации смертей, Росстат.

\section{ЗАКЛЮЧЕНИЕ}

Данное исследование вносит вклад в существующий задел методологических разработок, посвященных оценке потерь от транспортного травматизма, поскольку в рамках него:

1) предложен метод оценки потерь, связанных со смертностью (без использования внешней информации и модельных стандартов);

2) изучена возможность определения тяжести полученных травм с использованием информации об их локализации по МКБ-10.

Для более точной оценки требуется подробная детализация статистической информации, в частности показателей травматизма в ДТП в рамках формы №57 по возрасту, как и разработка статистических показателей по другим формам травматизма (в результате самоубийств, падений и др.). 


\section{БЛАГОДАРНОСТИ}

Автор выражает благодарность Евгению Михайловичу Андрееву за консультирование и помощь при подготовке статьи.

\section{ЛИТЕРАТУРА}

Murray C.J.L., A.D. Lopez (1997). Global mortality, disability, and the contribution of risk factors: Global Burden of Disease Study // The Lancet. 349, 9063: 1436-1442.

WHO, Regional office for Europe (1981). The epidemiology of accident traumas and resulting disabilities. Report on WHO Symposium: 45.

The World Bank (2014). Global Road Safety Facility, Transport for Health: The Global Burden of Disease from Motorized Road Transport. Washington, DC. 


\section{INTEGRAL INDICATORS OF DEMOGRAPHIC LOSSES FROM DEATHS AND INJURIES DUE TO ROAD ACCIDENTS IN RUSSIA *}

\section{ALEKSANDR RAMONOV}

Aleksandr Ramonov. National ReSEARCh University Higher School of Economics (Russia).

E-MAIL: aramonov@hse.ru.

DATE RECEIVED: October 2015 .

The article analyzes the dynamics and structure of deaths and injuries from road traffic accidents in Russia during 2006 to 2011. The analysis is based on the use of integrated indicators of years of life lost. It is shown that the maximum number of years of life lost due to excess mortality and disability (or gained from their elimination) is observed for men in the age group from 40 to 65 years, and for women between 55 and 75 years. New metrics of disability weights are developed.

Key words: external causes of death, health summary measures, road traffic accidents.

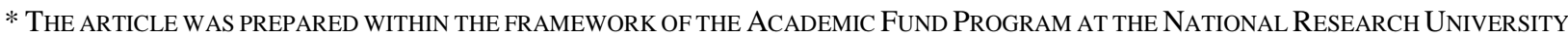
HIGHER SCHOOL OF ECONOMICS (HSE) IN 2015 (GRANT № 15-01-0032) AND SUPPORTED WITHIN THE FRAMEWORK OF A SUBSIDY GRANTED TO THE HSE BY THE GOVERNMENT OF THE RUSSIAN FEDERATION FOR THE IMPLEMENTATION OF THE GLOBAL COMPETITIVENESS PROGRAM.
}

\section{REFERENCES}

Murray C.J.L., A.D. Lopez (1997). Global mortality, disability, and the contribution of risk factors: Global Burden of Disease Study // The Lancet. 349, 9063: 1436-1442.

WHO, Regional office for Europe (1981). The epidemiology of accident traumas and resulting disabilities. Report on WHO Symposium: 45.

The World Bank (2014). Global Road Safety Facility, Transport for Health: The Global Burden of Disease from Motorized Road Transport. Washington, DC. 